Mots. Les langages du politique

$121 \mid 2019$

Restons groupés ! La construction discursive des relations sociales

\title{
Délier les langues sans défaire les liens
}

Helping tongues untie without undoing social ties

Abrir las bocas sin deshacer los lazos

Valérie Bonnet, Chloé Gaboriaux et Marie Plassart

\section{OpenEdition \\ Journals}

Édition électronique

URL : https://journals.openedition.org/mots/25544

DOI : $10.4000 /$ mots. 25544

ISSN : 1960-6001

Éditeur

ENS Éditions

Édition imprimée

Date de publication : 14 novembre 2019

Pagination : 9-14

ISBN : 979-10-362-0195-0

ISSN : 0243-6450

\section{Référence électronique}

Valérie Bonnet, Chloé Gaboriaux et Marie Plassart, « Délier les langues sans défaire les liens », Mots Les langages du politique [En ligne], 121 | 2019, mis en ligne le 01 janvier 2021, consulté le 23 avril

2022. URL : http://journals.openedition.org/mots/25544 ; DOI : https://doi.org/10.4000/mots.25544

\section{(c) ENS Éditions}




\section{Délier les langues sans défaire les liens}

«Restons groupés! L'injonction semble parfois vaine, parfois dangereuse. Quels qu'en soient les effets, elle reflète le travail social de création, d'entretien et de mise en cause des collectifs et des discours sur ces collectifs. Que signifie « appartenir à un groupe » lorsque la société est travaillée par des logiques individualistes tout autant que par le renouvellement des modalités de l'engagement collectif et la composition/recomposition des groupes? Les discours jouent à cet égard un rôle crucial : c'est à travers eux que les individus donnent du sens à leurs relations sociales, les font naître, exister, se transformer.

Parce que nous faisons l'hypothèse que la construction discursive des relations sociales emprunte des voies sinon analogues du moins comparables quels que soient les ensembles sociaux considérés, nous comprenons ici groupe dans un sens très large. Le mot peut désigner les ressortissants d'un État, dont la représentation en discours est un enjeu majeur des luttes politiques. De fait, depuis la fin du xvıı' siècle, le transfert de légitimité qui s'est opéré du roi de droit divin à la «nation » et au «peuple» impose aux acteurs politiques de déterminer la communauté des citoyens à laquelle ils s'adressent et de préciser les liens qui la font tenir. Le présent dossier prolonge à cet égard un intérêt que les analystes des langages politiques portent depuis longtemps aux «figures de la vie communautaire» (Job, Job, 1980) et plus largement aux «mots de la nation» (Rémi-Giraud, Rétat éd., 1996).

Mais groupe peut aussi renvoyer à des collectifs non étatiques. La façon dont le langage fait exister sur la scène politique des groupes infra-étatiques (nous dirions plutôt «intermédiaires ») a ainsi intéressé l'analyse de discours dès ses débuts. Elle constitue en effet le fondement de nos démocraties représentatives, qui impliquent l'existence de «porte-parole». En s'exprimant au nom d'une fraction du corps social, ces derniers sont amenés à mettre en avant un ou plusieurs critères de regroupement - l'origine, la classe sociale, le genre, l'âge ou la génération, etc. - et contribuent dès lors à délimiter une

Université Toulouse 3-Paul Sabatier, LERASS

valerie.bonnet@free.fr

Université de Lyon, Sciences Po Lyon, Triangle (UMR 5206)

chloe.gaboriaux@sciencespo-lyon.fr

Université Lyon 2, Triangle (UMR 5206)

marie.plassart@univ-lyon2.fr 
communauté et à en cimenter les relations (Bourdieu, 1984). L'entreprise partisane, qui a fait l'objet du précédent numéro de la revue Mots. Les langages du politique, en est l'exemple par excellence, en tant qu'elle mène à son terme le processus d'institutionnalisation politique d'un collectif (Bacot, 2018 ; Bacot, Lecolle éd., 2019). Elle n'épuise cependant pas le sujet, qui englobe aussi des groupes intermédiaires pour lesquels la forme partisane n'est pas pertinente et qui porte également sur des communautés transétatiques («la communauté des croyants», "les $99 \%$ », etc.) ou supra-étatiques (les «citoyens de l'Union européenne»).

En termes méthodologiques, l'étude de la construction discursive des relations sociales s'appuie bien souvent sur les dénominations sociopolitiques, dont Maurice Tournier invitait à faire la "grammaire» (Tournier, 1981). Dans cette perspective, l'attention est portée sur les effets de sens produits par les catégorisations, et notamment sur les rapports d'inclusion et d'exclusion qu'elles établissent entre les collectifs. L'usage du terme peuple au xıx siècle en est un bon exemple : il met en tension le peuple-plebs et le peuple-populus (Julliard, 1997), à travers un débat où s'affrontent ceux qui, comme Michelet, pensent les classes populaires (plebs) comme le meilleur du peuple, et ceux qui, comme Thiers en 1850, préfèreraient exclure la «vile multitude» du corps électoral. La sémantique lexicale conduit aussi à s’interroger sur les processus d'assignation et d'autodésignation que soutiennent les différentes manières de nommer. La définition d'autrui est ainsi toujours une définition de soi, comme le montre bien un récent ouvrage consacré à la mise en mots des migrations (Calabrese, Veniard éd., 2018).

L'analyste est alors conduit à aller au-delà du lexique pour enquêter sur les dispositifs énonciatifs (Geffroy éd., 1985) ou sur la désignation des liens ou des sentiments d'appartenance qu'il s'agit de susciter ou de consolider (attachement viscéral ou intérêt bien entendu, respect des traditions ou désir de vivre ensemble, pour ne donner que quelques exemples à la fois courants et schématiques). Ce qui nous a frappées dans l'abondante bibliographie consacrée au sujet, c'est l'accent placé sur la force illocutoire des discours qui s'efforcent de délimiter des groupes et de les faire exister, l'immense majorité des travaux travaillant à dévoiler les stratégies politiques qui les sous-tendent et à en souligner les effets perlocutoires.

La spécificité du présent dossier est d'en explorer le versant opposé. Les discours étudiés cherchent moins à faire advenir un groupe qu'à renégocier de l'intérieur les termes d'un découpage qui leur est imposé de l'extérieur, ce qui, pour reprendre le vocabulaire de l'anthropologie, conduit le chercheur à adopter une posture émique plutôt qu'étique. Quoique étudiant des cas ancrés dans des temps, des espaces et des collectifs très différents, les articles qui suivent ont tous pour objet des discours qui contribuent non pas à fermer les groupes sur eux-mêmes, mais au contraire à mettre en doute leurs frontières ou à les 
retravailler (Mathieu et Roussel éd., 2019). Le dialogue entre les articles se tient à la croisée de l'identité revendiquée, de l'identité assignée et du jeu entre catégories reconnues collectivement et positionnements individuels (Avanza, Laferté, 2005). Le dossier dans son ensemble souligne ainsi la part de liberté que le langage donne à l'individu dans le rapport qu'il entretient à ses appartenances sociales. Ce dernier y est bien cet «être-frontière » décrit par Georg Simmel, un «être de liaison qui doit toujours séparer et qui ne peut relier sans avoir séparé » (Simmel, 1988 [1909], p. 166). La notion de «nouvelle ethnicité» établie par John J. Gumperz (Gumperz, 1989 [1971-1986]) prend ici toute sa pertinence : ne reposant pas sur les processus traditionnels de proximités géographiques ou professionnelles et de confirmation quotidienne, le groupe est envisagé comme relevant de stratégies conscientes et d'expressions volontaristes de différenciation.

Là doit sans doute être cherchée la raison du silence du dossier sur les "classes sociales», leurs dénominations assumées ou contestées, qui tient à l'absence de propositions de contribution sur le sujet. Impensable dans le champ universitaire européen il y a encore quelques années, il témoigne des mutations de la société comme des grilles de lecture qui permettent de la rendre intelligible, comme l'a bien montré François Dubet (Dubet, 2012). Les approches retenues par les auteurs de ce numéro sont en effet plus attentives aux rapports singuliers que chacun entretient avec ses groupes d'appartenance et qui peuvent évoluer. Le dossier permet ainsi d'aborder cette tension entre liaison et séparation dans le temps, non seulement parce que les contributions traitent de périodes variées, mais aussi parce qu'elles rendent compte de l'épaisseur mémorielle des discours axés sur les groupes.

Significativement, les articles consacrés au long xıxe siècle (1789-1914) se penchent sur des termes qui marquent le rapport au groupement politique supérieur qu'est l'État. Dans une perspective d'histoire des idées, Anna Plassart et Suzanne Forbes s'interrogent ainsi sur le terme nationality tel qu'il est employé tout au long du xıxe siècle dans l'enceinte parlementaire britannique. Le traitement quantitatif de leur corpus montre que ses usages ne sont pas étrangers au «principe des nationalités » qui s'impose alors en Europe, associant étroitement sentiment national et souveraineté politique dans le cadre de l'État-nation. Le retour au texte, dans une démarche interprétative plus qualitative, leur permet cependant de mettre en lumière la façon dont les parlementaires en reconfigurent le sens, pour le rendre plus adéquat aux enjeux propres au Royaume-Uni. Ils rendent dès lors possible l'émergence de « concepts alternatifs d'identité et d'allégeance à l'État» souvent méconnus par les historiens.

Le sentiment national est aussi au cœur de l'article de Marie Plassart, qui l'envisage quant à elle à partir de la critique pacifiste qu'en font les socialistes américains au début du xxe siècle. Ils s'efforcent alors de déconstruire le terme patriot, à travers lequel leurs adversaires rendent inséparables attachement au 
pays et entrée en guerre, civisme et bellicisme. Selon une démarche empruntée à l'analyse des controverses, l'étude de leurs textes d'intervention politique (presse, pamphlets, caricatures) met l'accent sur la difficulté à maintenir dans le temps un positionnement bientôt décrit par leurs opposants comme unamerican.

Centrés sur des groupes sociaux ou politiques intermédiaires, les articles suivants donnent quant à eux une large place à la subjectivité des acteurs amenés à s’identifier ou être identifiés à des collectifs, car, comme le souligne Dell H. Hymes, «l'identité implique identification, une croyance ou un désir d'être comme ce à quoi l'on s'identifie » (Hymes, 1984 [1973-1982], p. 154). Dans l'article de Thomas Liano, l'étude littéraire emprunte ainsi aux théories de l'énonciation pour analyser la «danse des pronoms » dans les textes que Jean Genet consacre aux Black Panthers dans les années 1970. Comment un Européen blanc, ayant de surcroît revendiqué jusqu'alors son irréductible marginalité, peut-il faire corps avec les Black Panthers? L'interrogation hante le "nous» genetien, entre trahison de soi et engagement pour Autrui.

Maïlys Kydjian adopte quant à elle une démarche sociologique pour interroger des personnes que leurs origines confrontent à des groupes auxquels elles ne s’identifient pas ou pas complètement : Algériens, harkis, pieds-noirs. Les noms collectifs (Lecolle, 2016 et 2019) sont ici contestés par les individus qu'ils désignent et qui leur opposent d'autres délimitations et d'autres dénominations. Leurs discours révèlent la complexité du travail de la mémoire, qui les assigne à une communauté tout en leur permettant de la mettre à distance. Celle-ci constitue le lieu d'articulation de l'identité personnelle/individuelle, l’identité de groupe (définie par les relations interpersonnelles réelles) et l'identité communautaire (qui transcende en principe les individus et les groupes existants dans le temps et dans l'espace). Développée par Maurice Halbwachs (Halbwachs, 1994 [1925] et 1997 [1950]), cette vision n'est pas sans convergence avec les travaux sur les représentations sociales (voir le travail de Jean Viaud, 2003) en ce sens que la mémoire collective unit les membres d'un groupe autour d'une histoire, au même titre que les représentations sociales unissent les individus autour d'une conception du monde (dont l'histoire fait partie). En mettant le passé en adéquation avec les nécessités du présent, les représentations communes le chargent de fonctions identitaires (Haas, 2002).

Le dispositif dont Amaia Errecart rend compte pour finir est plus complexe dans la mesure où son enquête auprès des salariés et des bénévoles d'Amnesty International s'inscrit elle-même dans la politique de cohésion menée par l'association. Les personnes enquêtées ne cherchent pas seulement à discuter les catégories qui servent à les désigner - salariés ou professionnels, bénévoles ou militants -, elles savent que ce faisant elles participent à la redéfinition du groupe que constitue Amnesty. Les statuts sociaux constituent en effet tout autant des attributs structuraux que des ressources interactionnelles mobili- 
sables, le collectif se construisant non pas sur les identités, mais sur le partage de ce que doit être l'autrui généralisé (Mead, 1963 [1932]). Les discours manifestent ici ce qui travaille de façon plus souterraine les énoncés étudiés dans les autres contributions : qualifier une relation sociale, c'est déjà la transformer, et, dans les cas peut-être singuliers traités dans ce dossier, la complexifier plutôt que la figer.

\section{Références}

Avanza Martina, LAferté Gilles, 2005, «Dépasser la “construction des identités"? Identification, image sociale, appartenance », Genèses, n 61, p. 134-152.

BAcot Paul, 2018, «Onomastique partisane», dans Rencontres avec Michel Offerlé, H. Michel, S. Lévêque, J.-B. Contamin éd., Vulaines-sur-Seine, Éditions du Croquant, p. 293-301.

BAcot Paul, Lecolle Michelle éd., 2019, Nom d'un parti! Pour une onomastique partisane [numéro thématique], Mots. Les langages du politique, nº 120.

Bourdieu Pierre, 1984, "La délégation et le fétichisme politique », Actes de la recherche en sciences sociales, nº 52-53, p. 49-55.

CALABReSe Laura, VeniaRd Marie éd., 2018, Penser les mots, dire la migration, Louvainla-Neuve, Academia, L'Harmattan.

DUBEt François, 2012, "Classes sociales et description de la société », Revue française de socio-économie, $\mathrm{n}^{\circ}$ 10, p. 259-264.

Gefrroy Annie éd., 1985, Le nous politique [numéro thématique], Mots, ${ }^{\circ} 10$.

GUM PERZ John J., 1989 [1971-1986], Engager la conversation : introduction à la sociolinguistique interactionnelle, M. Dartevelle, M. Gilbert et I. Joseph trad., Paris, Minuit. HAAS Valérie, 2002, «Approche psychosociale d'une reconstruction historique. Le cas vichyssois », Les cahiers internationaux de psychologie sociale, n ${ }^{5}$ 33, p. 32-45.

HalbWachs Maurice, 1997 [1950], La mémoire collective, G. Namer éd., Paris, Albin Michel.

- 1994 [1925], Les cadres sociaux de la mémoire, Paris, Albin Michel.

Hymes Dell H., 1984 [1973-1982], Vers la compétence de communication, F. Mugler trad., Paris, Hatier, Credif.

Јов André, Јов Béatrice, 1980, «Les figures de la vie communautaire dans les réalisations lexicales du nouveau discours espagnol », Mots, nº 1, p. 77-94.

JULLIARD Jacques, 1997, «Le peuple», dans Les lieux de mémoire, P. Nora éd., Paris, Gallimard, vol. II, p. 2359-2393.

LECOLLE Michelle, 2019, Les noms collectifs humains en français : enjeux sémantiques, lexicaux et discursifs, Limoges, Lambert-Lucas.

- 2016, "Noms collectifs humains : nomination et prédication», Argumentation et analyse du discours, $n^{\circ}$ 17, http://aad.revues.org/2208 (consulté le 24 juin 2019).

Mathieu Lilian, Roussel Violaine éd., 2019, Penser les frontières sociales : enquêtes sur la culture, l'engagement et la politique, Lyon, Presses universitaires de Lyon. 
MeAd George H., 1963 [1932], L'esprit, le soi et la société, J. Cazeneuve, E. F. Kaelin et G. Thibault trad., Paris, PUF.

RÉMI-GIRAud Sylvianne, RÉTAT Pierre éd., 1996, Les mots de la nation, Lyon, Presses universitaires de Lyon.

Simmel Georg, 1988 [1909], «Pont et porte», dans G. Simmel, La tragédie de la culture et autres essais, S. Cornille et P. Ivernel trad., Marseille, Paris, Rivages, p. 159-166.

TOURNIER Maurice, 1981, «Vers une grammaire des dénominations socio-politiques au début de la Troisième République (1879-1905)», Mots, n² 2, p. 51-72.

VIAUD Jean, 2003, "Mémoire collective, représentations sociales et pratiques sociales», Connexions, n80, p.13-30. 